BULL. AUSTRAL. MATH. SOC.

$35 \mathrm{Al} 5, \quad 35 \mathrm{R} 30 ， \quad 76 \mathrm{H}$

VOL. 32 (1985), 147-149

\title{
TIDAL MODELLING AS AN INVERSE PROBLEM
}

\section{Peter Christopher Micintosh}

Classical limited-area tidal models rely on generally inadequate data along open-sea boundaries. Further errors may accrue due to the use of approximate dynamics and numerical coastline discretization. High quality data in the model interior is used only for model verification. Such data may be explicitly included in a linear tidal model using a weighted variational technique developed by Bennett and McIntosh [1].. The weights are chosen to reflect relative model uncertainties. This technique is an example of an inverse method, and is summarized and discussed in Chapter 2. A brief sumary of the use of inverse methods in oceanography is presented in Chapter 1 .

The utility of the variational method is demonstrated by constructing a model of the barotropic lunar semi-diurnal tide in Bass strait which is consistent with the dynamics, boundary conditions and data. The model is formulated as a correction to a classical tidal model. In Chapter 3, the classical model is constructed using an approximate radiation condition on open-sea boundaries; the computational advantages of such a condition are discussed in Chapter 2. Numerical procedures used are standard. The model does not agree with high-quality verification data obtained in Bass strait. Errors in shallow water dynamics, coastal and open boundary conditions and the data are studied in detail. The dominant errors occur in the open and coastal boundary conditions: the former due to data inadequacy and the necessity of using an approximation to the exact radiation condition, and

Received 19 March 1985. Thesis submitted to Monash University, November 1984. Degree approved February 1985. Supervisors: Dr C.B. Fandry, Dr A.F. Bennett and Professor B.R. Morton.

Copyright Clearance Centre, Inc. Serial-fee code: 0004-9727/85 $\$ A 2.00+0.00$. 
the latter due to numerical discretization. The relative errors are one hundred per cent and thirty per cent respectively. Data errors are predominantly due to positional error between the data location and the nearest model grid point. Relative errors are as high as twenty per cent. Dynamical errors arise from many sources, but the relative error sum does not exceed fifteen per cent.

Chapter 4 presents the variational tidal model of Bass strait. It is shown how the minimum correction (in a weighted least-squares sense) may be made to the classical model open boundary forcing such that the solution is consistent with the data. This solution is borderline in the sense that open boundary condition and data residuals equal the error estimates. A less extreme solution is obtained by accepting residuals in the dynamics and coastal boundary condition.

It is shown in Chapter 5 that if the variational model weights are chosen to be inversely proportional to squared error estimates, then the resulting solution is the maximum likelihood solution for Gaussian white errors. Weights based on this result lead to a tidal model with dynamical, boundary condition and data residuals that are less than error estimates. The model is relatively insensitive to the choice of weights, different classical solutions and data errors.

The variational method provides a procedure for the assessment of the information content of observational arrays before any instrument is deployed. Arrays consisting only of tide gauges are considered in Chapter 6. It is shown that in an ideal channel the variational procedure endeavours to synthesize the free modes of an infinite channel. Hence instruments should be located to facilitate the resolution of these modes. In particular, tide gauges are best deployed along open boundaries where the amplitude of exponentially decaying Poincare waves is largest. It is shown that the same criteria applies to the design of observational arrays in Bass strait. Further work on array design including the desirability of arrays containing different types of instruments is suggested. 


\section{Reference}

[1] A.F. Bennett and P.C. McIntosh, "Open ocean modelling as an inverse problem: Tidal theory", J. Phys. Oceconogr., 12 (1982), 1004-1018.

Department of Mathematics,

Monash University,

Clayton, Victoria, 3168. 